\title{
Trainable Post-Processing Method To Reduce False Alarms In The Detection Of Small Blotches Of Archive Films
}

\author{
Attila Licsár, László Czúni \\ Department of Image Processing and Neurocomputing \\ University of Veszprém \\ Veszprém, Hungary \\ \{licsara, czuni\}@almos.vein.hu
}

\author{
Tamás Szirányi \\ Analogical \& Neural Computing Laboratory \\ Hungarian Academy of Sciences \\ Budapest, Hungary \\ sziranyi@sztaki.hu
}

\begin{abstract}
We have developed a new semi-automatic neural network based method to detect blotches with low false alarm rate on archive films. Blotches can be modeled as temporal intensity discontinuities, hence false detection results originate from object motion (e.g. occlusion), non-rigid objects or erroneous motion estimation. In practice, usually, after the automatic detection step the false alarms are removed manually by an operator, significantly decreasing the efficiency of the restoration process. Our post-processing method classifies each detected blotch by its image features to minimize false results and the necessity of human intervention. The proposed method is tested on real archive sequences.
\end{abstract}

Keywords-digital film restoration; blotch detection; machine learning

\section{INTRODUCTION}

In national film archives there are huge amounts of archive films to be restored. These films suffer from several degradations such as blotches, scratches, flickering (intensity fluctuation), image vibration (displacement of adjacent frames), fading, discoloring, etc. Besides traditional analog techniques, semi-automatic digital restoration methods provide an efficient way to achieve cost efficient saving and reconstruction of the film heritage, i.e. fast, robust and automatic processing with a minimal human invention.

Some types of very annoying errors are called one frame defects and they are mostly visible as blotches. These artifacts appear at random positions on consecutive frames and with high contrast against the background. They have arbitrary shape, size and varying range of intensity (from bright to dark). Blotches are usually caused by dirt, damage of the film surface and chemical or biological processes such as mold. Blotches can be modeled as temporal discontinuities of pixel intensity not originating from object motion (occlusion, disocclusion) or non-rigid objects. A typical restoration procedure of one-frame defects is the following [9]: (1) detection of the defected regions, (2) interpolation of the corrupt image regions by spatio-temporal inpainting methods. In practice, after the automatic detection an operator manually verifies and corrects false results. In case of lots of false alarms the latter step is time consuming and results in a bottleneck of the restoration process. Our paper deals with an automatic detection step and with the minimization of the human intervention. The tuning of the detection parameters gives a trade-off between high correct detection and low false alarm rate (when an object is wrongly detected as artifact). In general, we prefer lower false alarm rate rather than high detection rate because the replacement of a real object with any inpainting, due to false detections, causes loss of original image details (e.g. buttons on the clothes) not acceptable by archivists. Hence an automatic method is needed to reduce false alarms of the previously detected blotches by classifying them. Serious problems are the influence of local/global motion and the presence of other film degradations such as vibration or flickering that yield false alarms in the detection phase. Further difficulty is the huge amount of data to be processed (e.g. motion estimation) at high resolution processing (2000x1500 pixels $(2 \mathrm{~K})$ or higher) of $35 \mathrm{~mm}$ archive films.

Our paper presents a blotch detection method with hierarchical gradient-based motion estimation with low computational cost. Optical flow calculation reduces false alarm detection rate owing to the object motion or image vibration. This step contains a preliminary detection step that speeds up the computation time of the optical flow by a preselection of the regions to be processed. Our main result is the improvement of detection efficiency during post-processing by a feature based neural network (NN) classification. This is essential to achieve a cost effective and efficient restoration by the reduction of the human intervention.

\section{OTHER WORKS}

Main approaches of blotch detection methods are in the following two groups: (1) detection by analysis of contrast or local maxima/minima; (2) methods based on the detection of temporal discontinuities. The first group includes morphological operator based methods [12],[7],[14],[4] resulting in low complexity because they do not require temporal analysis such as motion estimation. Methods in the second group are based on the detection of temporal discontinuities like the SDI (Spike Detection Index) [9], ROD (Rank Ordered Differences) [11][5], MRF (Markov Random Field) [8] methods. According to the comparative evaluation of 
these methods [11] ROD resulted in the highest accuracy while its computational cost was lower than MRF but higher than SDI.

A simplified ROD detector (SROD) was introduced in [13], which has lower complexity with approximately the same accuracy and moreover it comprises only one parameter. According to the temporal characteristics of blotches the SROD method analyses the maximal intensity difference between the actual pixels and the set of neighboring pixels on the preceding and consecutive images [6].

A common way to improve the detection accuracy of the temporal discontinuities is the analysis of motion compensated images of the previous and the following frames (bi-directional compensation). Methods usually apply block matching methods with multi-scale processing [2][13][11], and several heuristic searching (e.g. logarithmic) methods [5][2] to increase the computation speed. The drawbacks are that they do not guarantee optimal detection and the estimation gives only pixel accuracy. Our method works on 2-6K [3] resolution at reasonable speed. For this reason we have developed a modified hierarchical gradient-based method, which results sub-pixel accuracy and it is optimized for the fast detection of blotches.

\section{THE PROPOSED BLOTCH DETECTION METHOD}

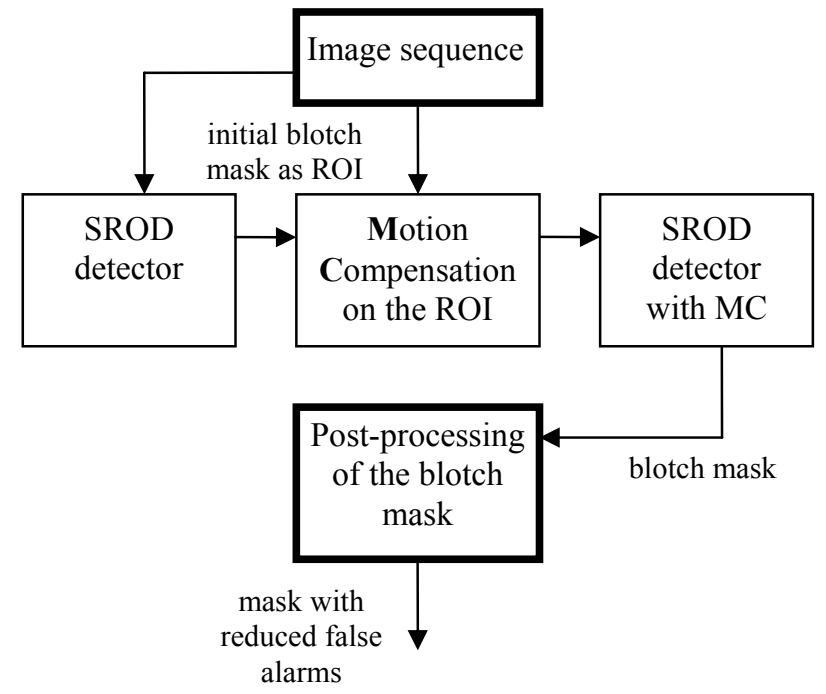

Fig. 1. Processing flow of the proposed blotch detection method.

Our aim is to accelerate the motion estimation and to decrease the false results on the detected blotch mask. The main steps of our algorithm are the following (see Fig. 1):

1. Initial detection: detection of the blotch mask by the low complexity SROD method without motion compensation. This operation serves high detection rate but also high false alarm rate due to uncompensated motions. Hence following steps aim at the decreasing of the false alarm results by filtering the detected blotches.

2. Main detection step: SROD detection with bidirectional motion compensation. This step reduces false alarms caused by object motion hence the motion estimation and compensation are done only on the initial blotch mask as ROI (region of interest).

3. Post-processing step: a neural network based classification method investigates the features of the defected image regions and omits residual false detections originating from the erroneous motion estimation caused by complex motion or object occlusion problem.

\section{A. The blotch detector and the motion compensation}

At least three frames are necessary to find one-frame defects so estimation is calculated with bi-directional motion compensation between the actual and neighboring images. We use a hierarchical gradient-based motion estimation method [1] that is based on Horn and Schunck's [6] optical flow constraint equations where the motion is modeled by simple translations. The applied constraints assume that pixel motion varies smoothly and continuously hence the frame-to-frame displacement is relatively small. To overcome this problem the method of Bergen [1] uses a multi-scale technique, employing a pyramid of successively low passed versions of the images. In this pyramid one finer image level is geometrically warped with bilinear interpolation by the scaled motion vectors of the previous level. Then the motion estimation on this warped level gives the residual finer motion resulting in a coarse-to-fine motion detection mechanism and this way we generate the motion compensated (MC) image at the same time.

The bi-directional optical flow calculation and the motion compensation of neighboring images are only processed in the predefined ROI of the initial SROD detection. The increase of the computation speed depends on the amount of object motions that in our experiments was a factor of two. Our blotch detection method takes about 10 seconds on a standard $1.7 \mathrm{Ghz}$ $\mathrm{PC}$ on $2 \mathrm{~K}$ resolution. The problem with the gradient-based motion estimation method is that it fails in the presence of temporal intensity variation (flicker), which is very common in case of archive films. Hence, we have to take into consideration the brightness variation and compensate it before the optical flow calculation. An efficient and robust flicker correction is implemented in [13]; the compensation parameters (1) of the linear flicker model (multiplicative and additive noise parameters) are calculated from the first order moments (mean: E[.], variance: $\operatorname{Var}[]$.$) in a given block (B) corresponding to the$ least mean square solution of the problem:

$$
\begin{aligned}
& a=\sqrt{\frac{\operatorname{Var}\left[B_{t-1}\right]}{\operatorname{Var}\left[B_{t}\right]}}, b=E\left[B_{t-1}\right]-a \cdot E\left[B_{t}\right], \\
& B_{t}^{\prime}(x, y)=a \cdot B_{t}(x, y)+b,
\end{aligned}
$$

where $a$ and $b$ are the compensation parameters of block B to calculate corrected intensity values in coordinate points $(\mathrm{x}, \mathrm{y})$. The solution of the low variance and local motion is detailed in [13]. For more accurate detection the block positions between adjacent frames are compensated by global motion parameters [10]. 


\section{B. Post-processing method by neural network}

Several post-processing methods are implemented in [13], such as hysteresis thresholding or constrained region growing of blotches to complete partially detected blotches. These methods analyze only the probability mask of the detector. Our new approach is a blotch-analyzing step where the brightness (luminance channel) of the image, localized by the mask, is analyzed and classified as blotch or as real object (non blotch). This classification is done by a feed-forward neural network trained by the image features of the detected blotches.

Our supervised training is divided into two phases: (1) detection and displaying of the blotches by the previously described automatic method in the selected frame for training purposes; (2) operator selects typical examples of positive (detected and displayed blotch is a real artifact) and negative (displayed blotch is not an artifact) samples by framing them with a rectangle. Features used to teach the neural network are as follows: the maximal horizontal and vertical intensity change inside the blotch area, local internal intensity contrast inside the blotch area, local internal mean and variance of the blotch area, and 3 parameters from the pixels neighboring the blotch candidate: external local contrast, mean and variance.

These parameters can distinguish real artifacts from false alarm objects, which are caused by erroneous motion estimation in case of small bright/dark objects (e.g. buttons on clothes) or fast moving objects, due to the motion blur or nonrigid motion. The human operator trains the system with $20-$ 30 samples from $2-4$ frames. After the back-propagation training of the network the blotches are detected automatically by the proposed method with neural network post filtering on the whole sequence. If the result is not satisfactory, the operator can expand the training set with new samples or rebuild the whole training set. This post-processing can reduce the false detection rate significantly. We tested a feed-forward neural network with back-propagation learning and found an optimal setup of the network configuration as follows: 6 inputs with extracted image features, 1 output with result of the decision and with 2 hidden layers with 6 and 4 neurons.

\section{EXPERIMENTAL RESULTS}

The objective evaluation of detection methods are done by the Receiver Operating Characteristic (ROC) curve (Fig. 2) that summarizes the probabilities of the correct detection and false alarm rates measuring it with several detection parameters.

Usually, the performance of other detection methods is statistically evaluated on artificially generated test sequences. Randomly selected image regions are replaced with a rendered blotch defined by a simple blotch model, i.e. homogenous blotch with sharp contour. On the contrary, our method is tested on real archive sequences so the ground truth data set of real artifacts is produced by manually marking blotches on real archive films. This is important because our post-processing method analyzes the intensity information of the detected regions and in case of artificially generated blotches the applied blotch model determines these. The test sequences are from the first Hungarian color film "Mattie the Goose-Boy". In our example two sequences (marked as $A$ and $B$ ) were tested after the operator trained them by samples from the two sequences.
We compared our proposed method of post-processing and the method based on the SROD detector of motion compensation. In the first test configuration the system was trained on sequence $A$ and then tested with both sequences while in our second test the system was trained on $B$ by the operator. It can be observed that if the training samples are from the same shot as the testing sequence then our proposed method results lower false alarms (if the image contains lots of false alarm to be reduced). If the training samples and test images are from distinct sequences then the improvement is not guaranteed (see the bottom graphs on Fig. 2). Fig. 2 shows the test results on the ROC curves where samples were trained with the $A$ and $B$ sequences. The training set involved about 10-20 manual selections and the method was tested on about 200 blotches.
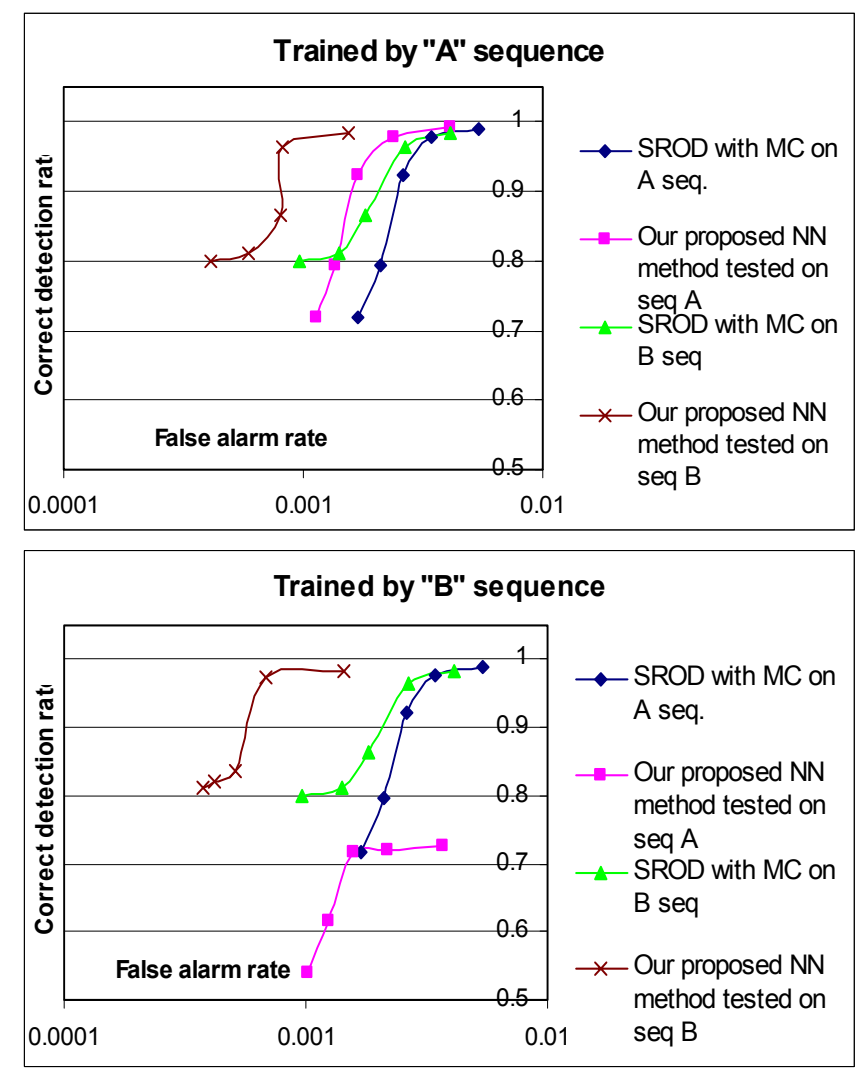

Fig. 2. Receiver Operating Characteristic (ROC) curve: performance evaluation of the standard SROD and our proposed method on A and B sequences and trained by one of them.

In Fig. 3 there is an example of our classification method where circles and rectangles indicate the detected blotches and the radius of them illustrate the size of the blotches. Rectangles indicate that our classification method identified the blotch as real object otherwise it is recognized as valid blotch. You can see that the buttons of clothes were recognized correctly as not being a blotch. 


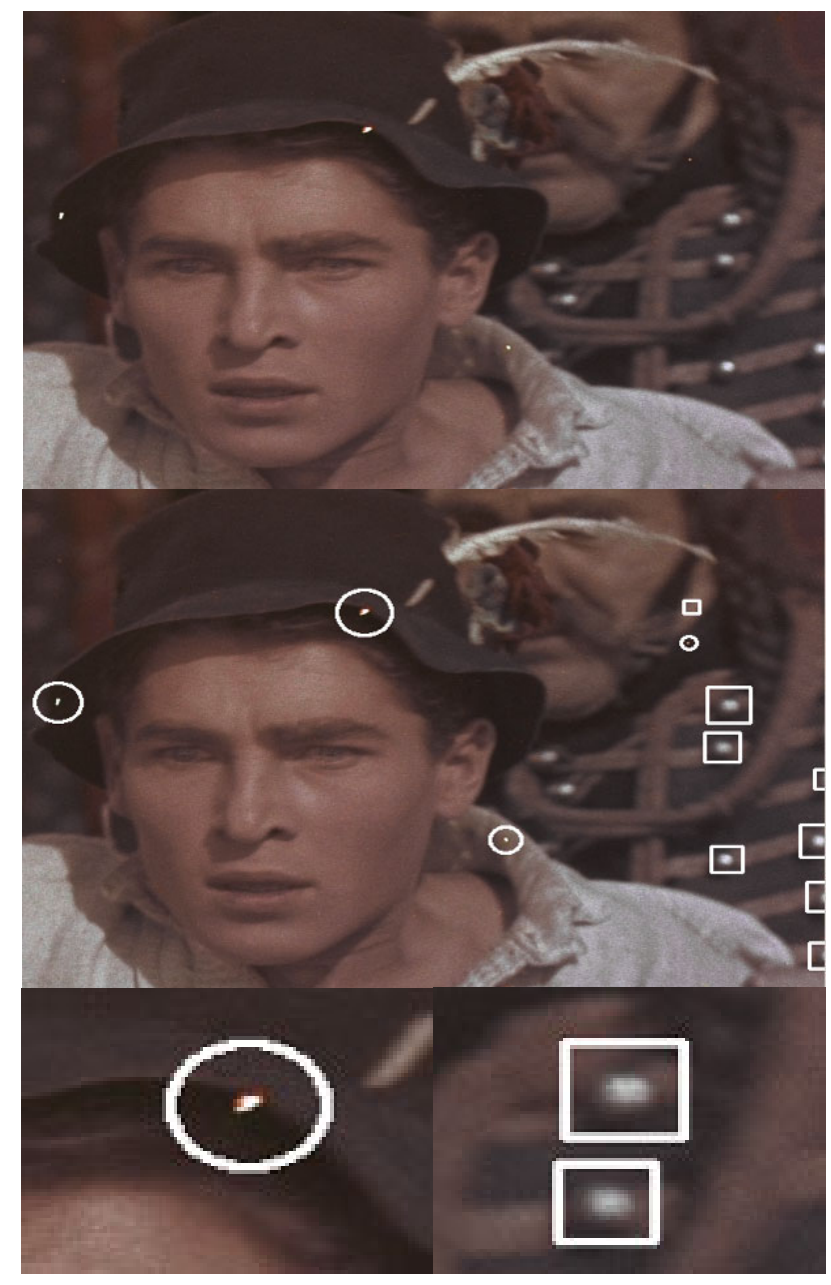

Fig. 3. Classification results of our post-processing method: original image (top), classification results (middle) and its zoomed image parts (bottom), where false alarms of the initial detections are marked with rectangles.

\section{CONCLUSION AND FUTURE WORKS}

We showed that our neural network based post-processing method significantly improves the detection efficiency by the automatic reduction of the false alarms without human intervention. Furthermore our optimized blotch detector speeds up the main detection phase and the motion estimation is invariant to flicker artifacts. These improvements make our method a cost effective blotch detection tool. In the future we aim to determine more consistent and robust blotch features for the classification and we intend to combine temporal methods with morphological operators to improve recognition efficiency.

\section{ACKNOWLEDGMENT}

Thanks to the Hungarian Film Archive for the scanned films. The project was supported by the DIMORF project of Hungarian National Research Program and the NoE MUSCLE project of Eu.

\section{REFERENCES}

[1] J.R. Bergen, and R. Hingorani, "Hierarchical Motion-Based Frame Rate Conversion," Technical Report, David Sarnoff Research Center, Princeton, 1990.

[2] O. Buisson, S. Boukir, and B. Besserer, "Motion compensated film restoration," Machine Vision and Applications, Springer-Verlag, vol. 13 (4), pp. 201-212, 2003.

[3] L. Czúni, A. Hanis, L. Kovács, B. Kránicz, A. Licsár, T. Szirányi, I. Kas, Gy. Kovács, and S. Manno, "Digital Motion Picture Restoration System for Film Archives (DIMORF)," SMPTE Motion Imaging Journal, Vol. 113, pp. 170-176, May-June 2004.

[4] L. Czúni, G. Császár, A. Hanis, L. Kovács, A. Licsár and T. Szirányi, "Semi Automatic Digital Motion Picture Restoration System with Learning Capabilities," Learning for Adaptable Visual Systems (LAVS), Cambridge, UK, 2004.

[5] A. Gangal, T. Kayikcioglu, and B. Dizdaroglu, "An improved motioncompensated restoration method for damage color motion picture film," Signal Proc.: Image Communication, vol. 19, pp. 353-368, 2004.

[6] B.K.P. Horn and BC. Schunk, "Determining Optical Flow," Artificial Intelligence, vol. 17, pp. 185-203, 1981.

[7] L. Joyeux, S.Boukir, B.Besserer, and O.Buisson, "Reconstruction of degraded image sequences. Application to film restoration," Image and Vision Computing (IVC) journal, Elsevier, Vol 19 (8), pp. 503-516, May 2001.

[8] A.C. Kokaram, R. Morris, W. Fitzgerald, and P. Rayner, "Detection of missing data in image sequences," IEEE Image Processing, pp. 1496$1508,1995$.

[9] A.C. Kokaram, Motion Picture Restoration: Digital Algorithms for Artefact Suppression in Degraded Motion Picture Film and Video. Springer Verlag, 1998.

[10] A. Licsár, L. Czúni, and T. Szirányi, "Adaptive Stabilization of Vibration on Archive Films," Lecture Notes in C.S, Springer-Verlag, LNCS 2756, pp. $230-237,2003$.

[11] M. J. Nadenau and S. K. Mitra, "Blotch and scratch detection in image sequences based on rank ordered differences," Time-Varying Image Processing and Moving Object Recognition, Elsevier, pp. 27-35, 1997.

[12] V. Naranjo, A. Albiol, J.M. Mossi, and A. Albiol, "Morphological Lambda Reconstruction applied to restoration of blotches in old films," Proc. of the 4th IASTED Intern. Conf. on Visualisation, Imaging and Image Processing, Spain, 2004.

[13] P. M. B. van Roosmalen, J. Biemond, and R. L. Lagendijk, "Restoration and storage of film and video archive material," Signal Processing for Multimedia, 1999.

[14] L. Tenze, G. Ramponi, and S. Carrato. "Blotches correction and contrast enhancement for old film pictures," Proc. Intern. Conf. on Image Processing, ICIP, Canada, pp. 660-663, 2000. 